\title{
The Relationship Between the Radiation Dose of Different Anatomic Bony Sites and Neutrophil Toxicity in Concurrent Chemoradiotherapy for Cervical Cancer
}

\author{
Baozhong Zhang ( $\square$ baozhongtj@163.com ) \\ Center for Cancer Research https://orcid.org/0000-0003-2445-4632
}

\section{Liming Xu}

Tianjin Medcical University Cancer Institute and Cancer Hospital, National Clinical Research Center for Cancer, Key Laboratory of Cancer prevention and therapy, Tianjin's Clinical Research Center for Cancer, Tianjin, China

\section{Zhiyan Liu}

Tianjin Medical University Cancer Institute and Hospital, National Clinical Research Center for Cancer, Key Laboratory of Cancer Prevention and Therapy, Tianjin's Clinical Research Center for Cancer, Tianjin, Chian

\section{Yanlan Chai}

Tianjin Medical University Cancer Institute and Hospital, National Clinical Research Center for Cancer, Key Laboratory of Cancer prevention and Therapy, Tianjin's Clinical Research Center for Cancer, Tianjin, China

\section{Yuanjie Cao}

Tianjin Medical University Cancer Institute and Hospital, National Clinical Research Center for Cancer, Key Laboratory of Cancer Prevention and Therapy, Tianjin's Clinical Research center for Cancer, Tianjin, China

\section{Jing Wang}

Tianjin Medical University Cancer Institute and Hospital, National Clinical Research Center for Cancer, Key Laboratory of Cancer Prevention and Therapy, Tianjin's Clinical Research Center for Cancer, Tianjin, China

\section{Hailing Hou}

Tianjin Medical University Cancer Institute and Hospital, National Clinical Research Center for Cancer, Key Laboratory of Cancer Prevention and Therapy, Tianjin's Clinical Research Center for Cancer, Tianjin, China

\section{Research article}

Keywords: Cervical cancer, Concurrent chemoradiotherapy, Intensity-modulated radiation therapy (IMRT), Neutrophiltoxicity, Dosimetric parameters

Posted Date: May 7th, 2020

DOI: https://doi.org/10.21203/rs.3.rs-26409/v1

License: (9) This work is licensed under a Creative Commons Attribution 4.0 International License. Read Full License 


\section{Abstract}

Background: The purpose of this study was to verify the radiation dose of the pelvic bone marrow of different anatomical bony sitesand the incidence of neutrophil toxicity during the concurrent chemoradiotherapy for cervical cancer.

Methods: There were 117 cervical cancer patients who received concurrent chemoradiotherapy included in this research. The radiotherapy includedexternal-beam radiation therapy (EBRT) and the brachytherapy. The dosimetric parameters included V5, V10, V20, V30, V40, V50, and Dmean. The final neutrophil count was defined as the lowest neutrophil count after 2 circles of concurrent chemotherapy, during or within 1 month after the treatment. The correlation between the dosimetric parameters and the lowest neutrophil count were analyzed by linear regression, the cutoff values of the dosimetric parameters were obtained using the ROC curves,the patients were divided into subgroups based on the cutoff values. The clinicopathologic features and the dosimetric parameters were included into the multivariate regression analysis model to further prove the correlation between the dosimetric parameters and the neutrophil toxicity. Data were assessed with IBM-SPSS software version 22.0, and all values of $p<0.05 w e r e$ considered statistically significant.

Results:The neutrophil toxicity (grade 1-4) rate was 58.97\%. The linear regression showed the Dmean and V50 of Lumbosacral vertebrae(LS), the Dmean, V5, V10, V20, V30, V40 and V50 of the ilium correlated with the lowest neutrophil count, while none of the dosimetric parameters of the femoral correlated with the lowest neutrophil count. The multivariate analysis showed the V20, V30 and V50 of the LS, the Dmean, V5, V10, V20 and V30 of the ilium correlated well with the neutrophil toxicity; none of the dosimetric parameters of the femoral correlated with the neutrophil toxicity.

Conclusion:During the process of concurrent chemoradiotherapy for cervical cancer, the volume of medium and high dose of LS and the volume of low and medium dose of ilium should be strictly limitedto reduce the risk of neutrophil toxicity, the Dmean of the ilium should also be taken into consideration. The dosimetric parameters of the femur could be ignored.

Trial registration:This is a retrospective research, and it will be retrospectively registered.

\section{Introduction:}

Cervical cancer is a most common gynecological malignancy, it ranks the third most common malignancy in females in the world ${ }^{[1-}$

2]. In China, there are 65,000 new cases of cervical cancer and 25,000 cervical cancer deaths every year ${ }^{[3]}$. Concurrent chemoradiotherapy has become the standard treatment for locally advanced cervical cancer ${ }^{[4]}$. The larger volume RT techniques have recently fallen out of favor, The use of Intensity-modulated radiation therapy (IMRT) technology and volume of intensitymodulated technology (Vmat) have effectively reduced the volume and dose of irradiation of the normal tissue, but low dose irradiation volume did not decrease ${ }^{[5,6]}$. In the process of concurrent chemoradiotherapy, hematologic toxicity is still a common clinical problem, while the neutrophil toxicity is the most common in all of the hematologic adverse events. When the neutrophil toxicity happens, it increases the risk of fever and infection, and sometimes, it might affect the intensity and progress of radiotherapy and chemotherapy, which may ultimately lead to poor outcome ${ }^{[7-9]}$. There have been many studies that proved the risk of hematologic adverse events correlated with the high dose and volume of pelvic bone marrow, and almost authors suggested to limit the dose and volume of bone marrow irradiation to reduce the risk of hematologic adverse events ${ }^{[10,11]}$. Several investigators have begun to consider the amount and distribution of low-dose radiation to the bone marrow as a part of their IMRT optimization process, so-called bone marrow-sparing IMRT. ${ }^{[12-16]}$ However, the hematopoietic function of bones depends on the content of their active bone marrow. Researchers have already proved the proportion of proliferating bone marrow is different in different anatomical sites of bones. ${ }^{[17-19]}$ The radiation dose of different anatomical skeletal sites might influence the hematologic toxicity differently. Therefore, we retrospectively analyzed 117 cervical cancer patients who received concurrent chemoradiotherapy, to prove the radiation dose of different anatomical sites in the pelvic radiotherapy influence the neutrophil toxicity in varying degree.

\section{Material And Methods}

\section{Patients}


We retrospectively analyzed the patients who received concurrent chemoradiotherapy between January 2016 to December 2018 in Tianjin Medical University Cancer Hospital, all of the patients were pathologically diagnosed cervical squamous carcinoma or adenocarcinoma, The inclusion criteria were: (1) staged IB-IIIC (based on Federation International of Gynecology and Obstetrics staging, Figo); (2) aged 18-70 years; (3) Karnofsky performance status score $\geq 70$; (4) complete records of blood routine examination at the time of pretreatment, weekly during the treatment and within a month after the treatment. The exclusion criteria were : (1) the concurrent chemotherapy finished less than 3 cycles; (2) the interrupt interval of radiotherapy longer than one week for any reason; (3) second primary tumor; (4) history of radiotherapy. There were 117 patients included into the study.

\section{Radiotherapy}

All of the patients received external-beam radiation therapy (EBRT) and the brachytherapy.

(1)

The EBRT: Radiotherapy was performed using the linear accelerator, $6 \mathrm{MV}-\mathrm{X}$ rays. Simulate patients prone with $\mathrm{CT}$ planning. The clinical target volume (CTV) includes gross tumor, entire uterine, cervix, vagina, the internal and external iliac lymph node drainage area, and the obturator lymph node drainage area, with a superior border at the lumbar $4 / 5$ level, and inferior border at $3 \mathrm{~cm}$ below the most inferior vaginal involvement; The gross tumor target volume of lymph node (GTVnd) includes all of the definitely diagnosed metastatic lymph nodes in pelvic cavity. The planning target volume of CTV (PTV) is to add $0.7 \mathrm{~cm}$ laterally and $1.5 \mathrm{~cm}$ axially on primary CTV, the planning target volume of GTVnd (PGTVnd) is to add $0.7 \mathrm{~cm}$ laterally and $1.5 \mathrm{~cm}$ axially on primary GTVnd. The total dose delivered to PGTVnd was 59.92 Gy(2.14 Gy per fraction, 28 fractions), the total dose delivered to PTV was 50.4 Gy(1.8 Gy per fraction, 28 fractions), and the radiation therapy progressed as 1 fraction/day 5 days/week. The contouring of organ at risk include small bowel, rectum, bladder and the femoral heads.

(2)

Brachytherapy: The brachytherapy was performed using Iridium-192, the brachytherapy started within 1 week after the EBRT, the total dose was $28 \mathrm{~Gy}$ (7Gy/fraction/week4weeks).

\section{The Concurrent Chemotherapy}

The concurrent chemotherapy regimen was cisplatin $\left(25 \mathrm{mg} / \mathrm{m}^{2}\right)$ weekly, started from the first week of the EBRT, totally 5 circles as planned. The chemotherapy was stopped when the WBC count was lower than $2.0 * 109 / \mathrm{L}$, or the neutrophil count was lower than $1.5^{*} 109 / \mathrm{L}$.

\section{Dosimetric Parameters Of The Bone Marrow}

The contouring of the bone marrow(BM) included the lower lumbosacral vertebrae (the superior border depending on the superior border of the PTV), the ilium and the upper femur(the inferior border depending on the inferior border of the PTV). The volume dosimetric parameters included V5(Volume receiving 5 Gy and so on), V10, V20, V30, V40, V50, and Dmean(the mean dose of the $\mathrm{BM})$. The effect of brachytherapy to BM was ignored.

\section{The Neutrophil Count}

The neutrophil was counted from the blood routine examination before, during and after the current chemoradiotherapy, the final neutrophil toxicity was defined as the lowest neutrophil count after 2 circles of concurrent chemotherapy, either during or within 1 month after the current chemoradiotherapy. And the neutrophil toxicity was defined from grade 0 to 4 as follows: grade 0 (normal), $\geq 2 \times 10^{9} / \mathrm{L}$; grade 1, 1.5-1.9 $\times 10^{9} / \mathrm{L} ;$ grade $2,1.0-1.4 \times 10^{9} / \mathrm{L} ;$ grade $3,0.5-0.9 \times 10^{9} / \mathrm{L} ;$ grade $4,<0.5 \times 10^{9} / \mathrm{L}$.

\section{The Statistical Analysis}


The correlation between the dosimetric parameters and the lowest neutrophil count were analyzed by linear regression, the cutoff values of the dosimetric parameters were obtained using the ROC curves. The patients were divided into subgroups based on the cutoff values of the dosimetric parameters. The clinicopathologic features and the dosimetric parameters were included into the multivariate regression analysis model to further prove the correlation between the dosimetric parameters and the neutrophil toxicity. Data were assessed with IBM-SPSS software version 22.0, and all values of $p<0.05$ were considered statistically significant.

\section{Results}

\section{The clinicopathologic features}

There were 117 patients included into the study, with a median age of $54(29-70)$ years, there were 83 cases with the age $<60$ years and 34 cases with the age $\geq 60$ years. Based on the pathology, there were 97 cases of Squamous carcinoma and 20 cases of adenocarcinoma. Based on the Figo stage, there were 9 cases of stage I, 79 cases of stage II and 29 cases of stage III. Based on the prescription radiation dose, there were 97 cases with the dose $<59.92 \mathrm{~Gy}$ and 20 cases with the dose $\geq 59.92 \mathrm{~Gy}$. Data was showed in Table 1. The total neutrophil toxicity (grade $1-4$ ) rate was $58.97 \%$; The age, pathology, stage and prescription dose were taken into the multivariate analysis.

Table 1

The clinicopathologic features of the patients

\begin{tabular}{|lr|}
\hline Clinicopathologic features & $\mathbf{n}$ \\
\hline Age & \\
\hline$<60 \mathrm{y}$ & 83 \\
$\geq \mathbf{6 0 y}$ & 34 \\
\hline Pathology & \\
\hline Squamous & 97 \\
\hline Adenocarcinoma & 20 \\
\hline Stage(Figo) & \\
\hline II (IIA-IIB) & 88 \\
\hline III (IIIA-IIIC) & 29 \\
\hline Prescription dose & 97 \\
\hline$<59.92$ Gy & 20 \\
\hline$\geq 59.92$ Gy
\end{tabular}

\section{The dosimetric parameters of the anatomical regions.}

The mean dose Dmean of the lumbosacral vertebrae(LS), ilium and femoral were $4284.68 \pm 35.39$ cGy, $3550.88 \pm 26.24$ cGy and $1547.06 \pm 46.81$ cGy respectively; the mean V 5 of the three regions were $374.83 \pm 83.27 \mathrm{ml}, 562.74 \pm 76.31 \mathrm{ml}$ and $204.08 \pm 54.49 \mathrm{ml}$ respectively; the mean $\mathrm{V} 10$ of the three regions were $372.52 \pm 83.03 \mathrm{ml}, 554.07 \pm 77.33 \mathrm{ml}$ and $134.83 \pm 57.22 \mathrm{ml}$ respectively; the mean V20 of the three regions were $356.51 \pm 82.71 \mathrm{ml}, 500.51 \pm 78.20 \mathrm{ml}$ and $66.46 \pm 51.13 \mathrm{ml}$ respectively; the mean V30 of the three regions were $312.40 \pm 81.96 \mathrm{ml}, 368.35 \pm 88.50 \mathrm{ml}$ and $29.74 \pm 28.43 \mathrm{ml}$ respectively; the mean $\mathrm{V} 40$ of the three regions were $252.76 \pm 76.14 \mathrm{ml}, 227.87 \pm 80.81 \mathrm{ml}$ and $6.10 \pm 6.63 \mathrm{ml}$ respectively; the mean V50 of the three regions were $141.09 \pm 49.97 \mathrm{ml}$, $97.11 \pm 53.54 \mathrm{ml}$ and $0.29 \pm 0.85 \mathrm{ml}$ respectively. The mean dose of the LS was higher than the other two regions, the volume of low and medium dose of the ilium was larger than the other two regions, the volume of high dose of the LS was higher than the other two regions. In the region of femoral, both of the mean dose and the volume of the low, medium and high dose were lower/smaller than the other two regions, while the V40 and V50 of the femoral were negligible compared with the other two regions.(Fig. 1-7) 


\section{The correlation between the dosimetric parameters and the lowest neutrophil count.}

The dosimetric parameters including Dmean, V5, V10, V20, V30, V40 and V50 of the three anatomical region namely lumbosacral vertebrae(LS), ilium and femoral were taken into the linear regression. The Dmean and V50 of LS, the Dmean, V5, V10, V20, V30, V40 and $\mathrm{V} 50$ of the ilium were found to correlate with the lowest neutrophil count, while none of the dosimetric parameters of the femoral correlated with the lowest neutrophil count. The results were showed in Table 2.

Table 2

The linear regression between the dosimetric parameters and the lowest neutrophil count.

\begin{tabular}{|llll|}
\hline & $\mathbf{F}$ & Adjusted R2 & $\mathbf{p}$ \\
\hline LSDmean & 8.087 & 0.421 & 0.035 \\
\hline IliacDmean & 13.147 & 0.612 & 0.000 \\
\hline FemurDmean & 1.698 & 0.215 & 0.195 \\
\hline LSV5 & 3.426 & 0.303 & 0.145 \\
\hline LSV10 & 3.339 & 0.272 & 0.274 \\
\hline LSV20 & 2.470 & 0.204 & 0.363 \\
\hline LSV30 & 1.818 & 0.188 & 0.311 \\
\hline LSV40 & 1.108 & 0.266 & 0.228 \\
\hline LSV50 & 5.274 & 0.356 & 0.000 \\
\hline IliacV5 & 10.937 & 0.579 & 0.001 \\
\hline IliacV10 & 9.277 & 0.467 & 0.003 \\
\hline IliacV20 & 11.555 & 0.683 & 0.001 \\
\hline IliacV30 & 13.018 & 0.694 & 0.000 \\
\hline IliacV40 & 20.478 & 0.744 & 0.000 \\
\hline IliacV50 & 21.301 & 0.749 & 0.000 \\
\hline FemurV5 & 5.720 & 0.339 & 0.118 \\
\hline FemurV10 & 0.658 & 0.103 & 0.419 \\
\hline FemurV20 & 0.202 & 0.007 & 0.654 \\
\hline FemurV30 & 1.623 & 0.005 & 0.205 \\
\hline FemurV40 & 0.036 & 0.008 & 0.851 \\
\hline FemurV50 & 0.016 & 0.009 & 0.899 \\
\hline
\end{tabular}

\section{The multivariate analysis for neutrophil toxicity.}

The patients were divided into subgroups based on the ROC lines for the the dosimetric parameters including Dmean, V5, V10, V20, V30, V40 and V50 of the LS, ilium and femoral respectively. Then all of the dosimetric parameters of the three regions were taken into the multivariate analysis for grade 1-4 neutrophil toxicity together with the age, pathology, stage, and prescription dose respectively. The V20, V30 and V50 of the LS correlated well with the neutrophil toxicity; the Dmean, V5, V10, V20 and V30 of the ilium correlated well with the neutrophil toxicity; none of the dosimetric parameters of the femoral correlated with the neutrophil 
toxicity. And the Figo stage(II) and the prescription dose were also correlated well with the neutrophil toxicity. The results were showed in Table 3 to 9.

Table 3

The multivariate analysis of Dmean of different anatomical bone regions for neutrophil toxicity

\begin{tabular}{|c|c|c|c|c|c|c|c|c|c|c|c|}
\hline & OR & $95 \% \mathrm{Cl}$ & $p$ & & OR & $95 \% \mathrm{Cl}$ & $p$ & & OR & $95 \% \mathrm{Cl}$ & $p$ \\
\hline Age & & & & Age & & & & Age & & & \\
\hline$<60$ & 1 & & & $<60$ & 1 & & & $<60$ & 1 & & \\
\hline$\geq 60$ & 0.723 & $\begin{array}{l}0.435- \\
1.202\end{array}$ & 0.212 & $\geq 60$ & 0.722 & $\begin{array}{l}0.435- \\
1.198\end{array}$ & 0.207 & $\geq 60$ & 0.736 & $\begin{array}{l}0.441- \\
1.227\end{array}$ & 0.240 \\
\hline Pathology & & & & Pathology & & & & Pathology & & & \\
\hline squamous & 1 & & & squamous & 1 & & & squamous & 1 & & \\
\hline Adeno & 1.615 & $\begin{array}{l}0.793- \\
3.286\end{array}$ & 0.186 & Adeno & 1.288 & $\begin{array}{l}0.629- \\
2.636\end{array}$ & 0.489 & Adeno & 1.573 & $\begin{array}{l}0.779- \\
3.176\end{array}$ & 0.207 \\
\hline Stage(Figo) & & & & Stage(Figo) & & & & Stage(Figo) & & & \\
\hline II & 1 & & & II & 1 & & & II & 1 & & \\
\hline III & 1.092 & $\begin{array}{l}0.610- \\
1.956\end{array}$ & 0.767 & III & 1.166 & $\begin{array}{l}0.656- \\
2.075\end{array}$ & 0.601 & III & 1.203 & $\begin{array}{l}0.673- \\
2.153\end{array}$ & 0.533 \\
\hline $\begin{array}{l}\text { The } \\
\text { prescription } \\
\text { dose }\end{array}$ & & & & $\begin{array}{l}\text { The } \\
\text { prescription } \\
\text { dose }\end{array}$ & & & & $\begin{array}{l}\text { The } \\
\text { prescription } \\
\text { dose }\end{array}$ & & & \\
\hline$<59.92$ Gy & 1 & & & $<59.92 \mathrm{~Gy}$ & 1 & & & $<59.92 \mathrm{~Gy}$ & 1 & & \\
\hline$\geq 59.92 \mathrm{~Gy}$ & 4.145 & $\begin{array}{l}1.650- \\
10.412\end{array}$ & 0.002 & $\geq 59.92$ Gy & 3.650 & $\begin{array}{l}1.455- \\
9.155\end{array}$ & 0.006 & $\geq 59.92$ Gy & 3.557 & $\begin{array}{l}1.413- \\
8.956\end{array}$ & 0.007 \\
\hline LSmean & & & & Iliacmean & & & & Femurmean & & & \\
\hline Group1 & 1 & & & Group1 & 1 & & & Group1 & 1 & & \\
\hline Group2 & 0.705 & $\begin{array}{l}0.430- \\
1.154\end{array}$ & 0.065 & Group2 & 1.462 & $\begin{array}{l}0.882- \\
2.424\end{array}$ & 0.041 & Group2 & 0.710 & 0.431 & 1.169 \\
\hline \multicolumn{4}{|c|}{ Group1: < 4039.85 cGy; } & \multicolumn{4}{|c|}{ Group1: < 3679.05 cGy; } & \multicolumn{4}{|c|}{ Group1: < 1547.06 cGy; } \\
\hline \multicolumn{4}{|c|}{ Group2: $\geq 4039.85$ cGy } & \multicolumn{4}{|c|}{ Group2: $\geq 3679.05$ cGy } & \multicolumn{4}{|c|}{ Group2: $\geq 1547.06$ cGy } \\
\hline
\end{tabular}


Table 4

The multivariate analysis of V5 of different anatomical bone regions for neutrophil toxicity

\begin{tabular}{|c|c|c|c|c|c|c|c|c|c|c|c|}
\hline & OR & $95 \% \mathrm{Cl}$ & $\mathbf{p}$ & & OR & $95 \% \mathrm{Cl}$ & $\mathbf{p}$ & & OR & $95 \% \mathrm{Cl}$ & $\mathbf{p}$ \\
\hline \multicolumn{4}{|l|}{ Age } & \multicolumn{4}{|l|}{ Age } & \multicolumn{4}{|l|}{ Age } \\
\hline$<60$ & 1 & & & $<60$ & 1 & & & $<60$ & 1 & & \\
\hline$\geq 60$ & 0.713 & $\begin{array}{l}0.427- \\
1.189\end{array}$ & 0.195 & $\geq 60$ & 0.646 & $\begin{array}{l}0.384- \\
1.087\end{array}$ & 0.100 & $\geq 60$ & 0.703 & $\begin{array}{l}0.416- \\
1.187\end{array}$ & 0.187 \\
\hline \multicolumn{4}{|l|}{ Pathology } & \multicolumn{4}{|l|}{ Pathology } & \multicolumn{4}{|l|}{ Pathology } \\
\hline Squamous & 1 & & & Squamous & 1 & & & Squamous & 1 & & \\
\hline Adeno & 1.611 & $\begin{array}{l}0.803- \\
3.231\end{array}$ & 0.180 & Adeno & 1.424 & $\begin{array}{l}0.703- \\
2.887\end{array}$ & 0.327 & Adeno & 1.422 & $\begin{array}{l}0.699- \\
2.891\end{array}$ & 0.331 \\
\hline \multicolumn{4}{|l|}{ Stage(Figo) } & \multicolumn{4}{|l|}{ Stage(Figo) } & \multicolumn{4}{|l|}{ Stage(Figo) } \\
\hline II & 1 & & & ॥ & 1 & & & II & 1 & & \\
\hline III & 1.200 & $\begin{array}{l}0.672- \\
2.145\end{array}$ & 0.537 & III & 1.123 & $\begin{array}{l}0.631- \\
2.000\end{array}$ & 0.693 & III & 1.148 & $\begin{array}{l}0.645- \\
2.044\end{array}$ & 0.639 \\
\hline \multicolumn{4}{|l|}{$\begin{array}{l}\text { The } \\
\text { prescription } \\
\text { dose }\end{array}$} & \multicolumn{4}{|l|}{$\begin{array}{l}\text { The } \\
\text { prescription } \\
\text { dose }\end{array}$} & \multicolumn{4}{|l|}{$\begin{array}{l}\text { The } \\
\text { prescription } \\
\text { dose }\end{array}$} \\
\hline$<59.92 \mathrm{~Gy}$ & 1 & & & $<59.92$ Gy & 1 & & & $<59.92$ Gy & 1 & & \\
\hline$\geq 59.92 \mathrm{~Gy}$ & 3.214 & $\begin{array}{l}1.259- \\
8.206\end{array}$ & 0.015 & $\geq 59.92$ Gy & 3.394 & $\begin{array}{l}1.342- \\
8.585\end{array}$ & 0.010 & $\geq 59.92$ Gy & 3.843 & $\begin{array}{l}1.534- \\
9.629\end{array}$ & 0.004 \\
\hline \multicolumn{4}{|l|}{ LSV5 } & \multicolumn{4}{|l|}{ IliacV5 } & \multicolumn{4}{|l|}{ FemurV5 } \\
\hline Group1 & 1 & & & Group1 & 1 & & & Group1 & 1 & & \\
\hline Group2 & 1.647 & $\begin{array}{l}0.997- \\
2.721\end{array}$ & 0.051 & Group2 & 1.664 & $\begin{array}{l}0.981- \\
2.821\end{array}$ & 0.039 & Group2 & 1.053 & $\begin{array}{l}0.642- \\
1.726\end{array}$ & 0.838 \\
\hline \multicolumn{4}{|c|}{ Group1: < 340.765 ml; } & \multicolumn{3}{|c|}{ Group1: > 517.500 ml; } & \multicolumn{3}{|c|}{ Group1: <183.04 ml; } & & \\
\hline \multicolumn{4}{|c|}{ Group2: $\geq 340.765 \mathrm{ml}$} & \multicolumn{3}{|c|}{ Group2: $\geq 517.500 \mathrm{ml}$} & \multicolumn{3}{|c|}{ Group2: $\geq 183.04 \mathrm{ml}$} & & \\
\hline
\end{tabular}


Table 5

The multivariate analysis of V10 of different anatomical bone regions for neutrophil toxicity

\begin{tabular}{|c|c|c|c|c|c|c|c|c|c|c|c|}
\hline & OR & $95 \% \mathrm{Cl}$ & p & & OR & $95 \% \mathrm{Cl}$ & $\mathrm{p}$ & & OR & $95 \% \mathrm{Cl}$ & p \\
\hline Age & & & & Age & & & & Age & & & \\
\hline$<60$ & 1 & & & $<60$ & 1 & & & $<60$ & 1 & & \\
\hline$\geq 60$ & 0.712 & $\begin{array}{l}0.426- \\
1.188\end{array}$ & 0.193 & $\geq 60$ & 0.646 & $\begin{array}{l}0.384- \\
1.088\end{array}$ & 0.100 & $\geq 60$ & 0.732 & $\begin{array}{l}0.435- \\
1.232\end{array}$ & 0.240 \\
\hline Pathology & & & & Pathology & & & & Pathology & & & \\
\hline Squamous & 1 & & & Squamous & 1 & & & Squamous & 1 & & \\
\hline Adeno & 1.629 & $\begin{array}{l}0.813- \\
3.264\end{array}$ & 0.169 & Adeno & 1.421 & $\begin{array}{l}0.701- \\
2.881\end{array}$ & 0.329 & Adeno & 1.497 & $\begin{array}{l}0.731- \\
3.063\end{array}$ & 0.270 \\
\hline Stage(Figo) & & & & Stage(Figo) & & & & Stage(Figo) & & & \\
\hline II & 1 & & & II & 1 & & 0.003 & II & 1 & & \\
\hline III & 1.210 & $\begin{array}{l}0.677- \\
2.162\end{array}$ & 0.520 & III & 1.123 & $\begin{array}{l}0.630- \\
2.000\end{array}$ & 0.694 & III & 1.164 & $\begin{array}{l}0.652- \\
2.078\end{array}$ & 0.607 \\
\hline $\begin{array}{l}\text { The } \\
\text { prescription } \\
\text { dose }\end{array}$ & & & & $\begin{array}{l}\text { The } \\
\text { prescription } \\
\text { dose }\end{array}$ & & & & $\begin{array}{l}\text { The } \\
\text { prescription } \\
\text { dose }\end{array}$ & & & \\
\hline$<59.92$ Gy & 1 & & & $<59.92$ Gy & 1 & & & $<59.92$ Gy & 1 & & \\
\hline$\geq 59.92 \mathrm{~Gy}$ & 3.159 & $\begin{array}{l}1.239- \\
8.057\end{array}$ & 0.016 & $\geq 59.92 \mathrm{~Gy}$ & 3.501 & $\begin{array}{l}1.390- \\
8.819\end{array}$ & 0.008 & $\geq 59.92$ Gy & 3.697 & $\begin{array}{l}1.453- \\
9.410\end{array}$ & 0.006 \\
\hline LSV10 & & & & IliacV10 & & & & FemurV10 & & & \\
\hline Group1 & 1 & & & Group1 & 1 & & & Group1 & 1 & & \\
\hline Group2 & 1.751 & $\begin{array}{l}1.058- \\
2.899\end{array}$ & 0.069 & Group2 & 1.632 & $\begin{array}{l}0.963- \\
2.763\end{array}$ & 0.029 & Group2 & 0.880 & $\begin{array}{l}0.531- \\
1.460\end{array}$ & 0.622 \\
\hline \multicolumn{4}{|c|}{ Group1: < $333.215 \mathrm{ml}$; } & \multicolumn{4}{|c|}{ Group1: < 500.645 ml; } & \multicolumn{4}{|c|}{ Group1: < 87.625 ml; } \\
\hline \multicolumn{4}{|c|}{ Group2: $\geq 333.215 \mathrm{ml}$} & \multicolumn{4}{|c|}{ Group2: $\geq 500.645 \mathrm{ml}$} & \multicolumn{4}{|c|}{ Group2: $\geq 87.625 \mathrm{ml}$} \\
\hline
\end{tabular}


Table 6

The multivariate analysis of V20 of different anatomical bone regions for neutrophil toxicity

\begin{tabular}{|c|c|c|c|c|c|c|c|c|c|c|c|}
\hline & OR & $95 \% \mathrm{Cl}$ & $p$ & & OR & $95 \% \mathrm{Cl}$ & $\mathrm{p}$ & & OR & $95 \% \mathrm{Cl}$ & p \\
\hline Age & & & & Age & & & & Age & & & \\
\hline$<60$ & 1 & & & $<60$ & 1 & & & $<60$ & 1 & & \\
\hline$\geq 60$ & 0.711 & $\begin{array}{l}0.426- \\
1.187\end{array}$ & 0.192 & $\geq 60$ & 0.687 & $\begin{array}{l}0.410- \\
1.153\end{array}$ & 0.155 & $\geq 60$ & 0.774 & $\begin{array}{l}0.465- \\
1.290\end{array}$ & 0.326 \\
\hline Pathology & & & & Pathology & & & & Pathology & & & \\
\hline Squamous & 1 & & & Squamous & 1 & & & Squamous & 1 & & \\
\hline Adeno & 1.561 & $\begin{array}{l}0.781- \\
3.122\end{array}$ & 0.208 & Adeno & 1.413 & $\begin{array}{l}0.699- \\
2.857\end{array}$ & 0.336 & Adeno & 1.875 & $\begin{array}{l}0.923- \\
3.810\end{array}$ & 0.082 \\
\hline Stage(Figo) & & & & Stage(Figo) & & & & Stage(Figo) & & & \\
\hline II & 1 & & & II & 1 & & & II & 1 & & \\
\hline III & 1.167 & $\begin{array}{l}0.655- \\
2.082\end{array}$ & 0.600 & III & 1.121 & $\begin{array}{l}0.628- \\
2.003\end{array}$ & 0.698 & III & 1.191 & $\begin{array}{l}0.669- \\
2.119\end{array}$ & 0.553 \\
\hline $\begin{array}{l}\text { The } \\
\text { prescription } \\
\text { dose }\end{array}$ & & & & $\begin{array}{l}\text { The } \\
\text { prescription } \\
\text { dose }\end{array}$ & & & & $\begin{array}{l}\text { The } \\
\text { prescription } \\
\text { dose }\end{array}$ & & & \\
\hline$<59.92$ Gy & 1 & & & $<59.92$ Gy & 1 & & & $<59.92$ Gy & 1 & & \\
\hline$\geq 59.92 \mathrm{~Gy}$ & 2.737 & $\begin{array}{l}1.018- \\
7.360\end{array}$ & 0.046 & $\geq 59.92 \mathrm{~Gy}$ & 3.707 & $\begin{array}{l}1.473- \\
9.327\end{array}$ & 0.005 & $\geq 59.92$ Gy & 3.735 & $\begin{array}{l}1.495- \\
9.336\end{array}$ & 0.005 \\
\hline LSV20 & & & & IliacV20 & & & & FemurV20 & & & \\
\hline Group1 & 1 & & & Group1 & 1 & & & Group1 & 1 & & \\
\hline Group2 & 1.745 & $\begin{array}{l}1.011- \\
3.012\end{array}$ & 0.046 & Group2 & 1.224 & $\begin{array}{l}0.736- \\
2.036\end{array}$ & 0.036 & Group2 & 0.459 & $\begin{array}{l}0.273- \\
0.771\end{array}$ & 0.503 \\
\hline \multicolumn{4}{|c|}{ Group1: < $326.70 \mathrm{ml}$; } & \multicolumn{3}{|c|}{ Group1: < 451.715 ml; } & \multicolumn{3}{|c|}{ Group1: < $64.595 \mathrm{ml}$; } & & \\
\hline \multicolumn{4}{|c|}{ Group2: $\geq 326.70 \mathrm{ml}$} & \multicolumn{3}{|c|}{ Group2: $\geq 451.715 \mathrm{ml}$} & \multicolumn{3}{|c|}{ Group2: $\geq 64.595 \mathrm{ml}$} & & \\
\hline
\end{tabular}


Table 7

The multivariate analysis of V30 of different anatomical bone regions for neutrophil toxicity

\begin{tabular}{|c|c|c|c|c|c|c|c|c|c|c|c|}
\hline & OR & $95 \% \mathrm{Cl}$ & $\mathbf{p}$ & & OR & $95 \% \mathrm{Cl}$ & $\mathbf{p}$ & & OR & $95 \% \mathrm{Cl}$ & $\mathbf{p}$ \\
\hline \multicolumn{4}{|l|}{ Age } & \multicolumn{4}{|l|}{ Age } & \multicolumn{4}{|l|}{ Age } \\
\hline$<60$ & 1 & & & $<60$ & 1 & & & $<60$ & 1 & & \\
\hline$\geq 60$ & 0.713 & $\begin{array}{l}0.428- \\
1.189\end{array}$ & 0.195 & $\geq 60$ & 0.691 & $\begin{array}{l}0.414- \\
1.151\end{array}$ & 0.156 & $\geq 60$ & 0.720 & $\begin{array}{l}0.431- \\
1.203\end{array}$ & 0.209 \\
\hline \multicolumn{4}{|l|}{ Pathology } & \multicolumn{4}{|l|}{ Pathology } & \multicolumn{4}{|l|}{ Pathology } \\
\hline Squamous & 1 & & & Squamous & 1 & & & Squamous & 1 & & \\
\hline Adeno & 1.503 & $\begin{array}{l}0.752- \\
3.002\end{array}$ & 0.249 & Adeno & 1.320 & $\begin{array}{l}0.640- \\
2.723\end{array}$ & 0.452 & Adeno & 1.668 & $\begin{array}{l}0.833- \\
3.420\end{array}$ & 0.146 \\
\hline \multicolumn{4}{|l|}{ Stage(Figo) } & \multicolumn{4}{|l|}{ Stage(Figo) } & \multicolumn{4}{|l|}{ Stage(Figo) } \\
\hline II & 1 & & & II & 1 & & & II & 1 & & \\
\hline III & 1.161 & $\begin{array}{l}0.652- \\
2.068\end{array}$ & 0.612 & III & 1.166 & $\begin{array}{l}0.655- \\
2.076\end{array}$ & 0.601 & III & 1.033 & $\begin{array}{l}0.577- \\
1.849\end{array}$ & 0.913 \\
\hline \multicolumn{4}{|l|}{$\begin{array}{l}\text { The } \\
\text { prescription } \\
\text { dose }\end{array}$} & \multicolumn{4}{|l|}{$\begin{array}{l}\text { The } \\
\text { prescription } \\
\text { dose }\end{array}$} & \multicolumn{4}{|l|}{$\begin{array}{l}\text { The } \\
\text { prescription } \\
\text { dose }\end{array}$} \\
\hline$<59.92 \mathrm{~Gy}$ & 1 & & & $<59.92$ Gy & 1 & & & $<59.92$ Gy & 1 & & \\
\hline$\geq 59.92 \mathrm{~Gy}$ & 3.161 & $\begin{array}{l}1.240- \\
8.060\end{array}$ & 0.016 & $\geq 59.92 \mathrm{~Gy}$ & 3.762 & $\begin{array}{l}1.503- \\
9.417\end{array}$ & 0.005 & $\geq 59.92$ Gy & 3.851 & $\begin{array}{l}1.540- \\
9.629\end{array}$ & 0.004 \\
\hline \multicolumn{4}{|l|}{ LSV30 } & \multicolumn{4}{|l|}{ IliacV30 } & \multicolumn{4}{|l|}{ FemurV30 } \\
\hline Group1 & 1 & & & Group1 & 1 & & & Group1 & 1 & & \\
\hline Group2 & 1.738 & $\begin{array}{l}1.032- \\
2.926\end{array}$ & 0.038 & Group2 & 1.321 & $\begin{array}{l}0.798- \\
2.187\end{array}$ & 0.029 & Group2 & 0.443 & $\begin{array}{l}0.263- \\
0.747\end{array}$ & 0.332 \\
\hline \multicolumn{4}{|c|}{ Group1: < 312.62 ml; } & \multicolumn{3}{|c|}{ Group1: < 288.475 ml; } & \multicolumn{3}{|c|}{ Group1: < $35.98 \mathrm{ml}$; } & & \\
\hline \multicolumn{4}{|c|}{ Group2: $\geq 312.62 \mathrm{ml}$} & \multicolumn{3}{|c|}{ Group2: $\geq 288.475 \mathrm{ml}$} & \multicolumn{3}{|c|}{ Group2: $\geq 35.98 \mathrm{ml}$} & & \\
\hline
\end{tabular}


Table 8

The multivariate analysis of V40 of different anatomical bone regions for neutrophil toxicity

\begin{tabular}{|c|c|c|c|c|c|c|c|c|c|c|c|}
\hline & OR & $95 \% \mathrm{Cl}$ & p & & OR & $95 \% \mathrm{Cl}$ & p & & OR & $95 \% \mathrm{Cl}$ & $\mathbf{p}$ \\
\hline \multicolumn{4}{|l|}{ Age } & \multicolumn{4}{|l|}{ Age } & \multicolumn{4}{|l|}{ Age } \\
\hline$<60$ & 1 & & & $<60$ & 1 & & & $<60$ & 1 & & \\
\hline$\geq 60$ & 0.711 & $\begin{array}{l}0.428- \\
1.182\end{array}$ & 0.189 & $\geq 60$ & 0.706 & $\begin{array}{l}0.425- \\
1.172\end{array}$ & 0.178 & $\geq 60$ & 0.714 & $\begin{array}{l}0.429- \\
1.186\end{array}$ & 0.193 \\
\hline \multicolumn{4}{|l|}{ Pathology } & \multicolumn{4}{|l|}{ Pathology } & \multicolumn{4}{|l|}{ Pathology } \\
\hline Squamous & 1 & & & Squamous & 1 & & & Squamous & 1 & & \\
\hline Adeno & 1.447 & $\begin{array}{l}0.719- \\
2.911\end{array}$ & 0.300 & Adeno & 1.259 & $\begin{array}{l}0.607- \\
2.613\end{array}$ & 0.535 & Adeno & 1.440 & $\begin{array}{l}0.714- \\
2.904\end{array}$ & 0.308 \\
\hline \multicolumn{4}{|l|}{ Stage(Figo) } & \multicolumn{4}{|l|}{ Stage(Figo) } & \multicolumn{4}{|l|}{ Stage(Figo) } \\
\hline II & 1 & & & II & 1 & & & II & 1 & & \\
\hline III & 1.163 & $\begin{array}{l}0.652- \\
2.073\end{array}$ & 0.610 & III & 1.136 & $\begin{array}{l}0.640- \\
2.017\end{array}$ & 0.663 & III & 1.143 & $\begin{array}{l}0.641- \\
2.037\end{array}$ & 0.651 \\
\hline \multicolumn{4}{|l|}{$\begin{array}{l}\text { The } \\
\text { prescription } \\
\text { dose }\end{array}$} & \multicolumn{4}{|l|}{$\begin{array}{l}\text { The } \\
\text { prescription } \\
\text { dose }\end{array}$} & \multicolumn{4}{|l|}{$\begin{array}{l}\text { The } \\
\text { prescription } \\
\text { dose }\end{array}$} \\
\hline$<59.92$ Gy & 1 & & & $<59.92$ Gy & 1 & & & $<59.92$ Gy & 1 & & \\
\hline$\geq 59.92 \mathrm{~Gy}$ & 3.764 & $\begin{array}{l}1.493- \\
9.492\end{array}$ & 0.005 & $\geq 59.92 \mathrm{~Gy}$ & 3.413 & $\begin{array}{l}1.355- \\
8.598\end{array}$ & 0.009 & $\geq 59.92$ Gy & 3.871 & $\begin{array}{l}1.550- \\
9.667\end{array}$ & 0.004 \\
\hline \multicolumn{4}{|l|}{ LSV40 } & \multicolumn{4}{|l|}{ IliacV40 } & \multicolumn{4}{|l|}{ FemurV40 } \\
\hline Group1 & 1 & & & Group1 & 1 & & & Group1 & 1 & & \\
\hline Group2 & 1.111 & $\begin{array}{l}0.685- \\
1.802\end{array}$ & 0.067 & Group2 & 1.621 & $\begin{array}{l}0.990- \\
2.654\end{array}$ & 0.055 & Group2 & 1.073 & $\begin{array}{l}0.648- \\
1.776\end{array}$ & 0.786 \\
\hline \multicolumn{4}{|c|}{ Group1: < 223.855 ml; } & \multicolumn{3}{|c|}{ Group1: < 160.930 ml; } & \multicolumn{3}{|c|}{ Group1: < $9.790 \mathrm{ml}$; } & & \\
\hline \multicolumn{4}{|c|}{ Group2: $\geq 223.855 \mathrm{ml}$} & \multicolumn{3}{|c|}{ Group2: $\geq 160.930 \mathrm{ml}$} & \multicolumn{3}{|c|}{ Group2: $\geq 9.790 \mathrm{ml}$} & & \\
\hline
\end{tabular}


Table 9

The multivariate analysis of V50 of different anatomical bone regions for neutrophil toxicity

\begin{tabular}{|c|c|c|c|c|c|c|c|c|c|c|c|}
\hline & OR & $95 \% \mathrm{Cl}$ & p & & OR & $95 \% \mathrm{Cl}$ & $p$ & & OR & $95 \% \mathrm{Cl}$ & p \\
\hline Age & & & & Age & & & & Age & & & \\
\hline$<60$ & 1 & & & $<60$ & 1 & & & $<60$ & 1 & & \\
\hline$\geq 60$ & 0.701 & $\begin{array}{l}0.422- \\
1.166\end{array}$ & 0.171 & $\geq 60$ & 0.715 & $\begin{array}{l}0.430- \\
1.188\end{array}$ & 0.195 & $\geq 60$ & 0.709 & $\begin{array}{l}0.425- \\
1.183\end{array}$ & 0.188 \\
\hline Pathology & & & & Pathology & & & & Pathology & & & \\
\hline Squamous & 1 & & & Squamous & 1 & & & Squamous & 1 & & \\
\hline Adeno & 1.277 & $\begin{array}{l}0.620- \\
2.629\end{array}$ & 0.507 & Adeno & 1.314 & $\begin{array}{l}0.639- \\
2.700\end{array}$ & 0.458 & Adeno & 1.444 & $\begin{array}{l}0.715- \\
2.914\end{array}$ & 0.306 \\
\hline Stage(Figo) & & & & Stage(Figo) & & & & Stage(Figo) & & & \\
\hline II & 1 & & & II & 1 & & & II & 1 & & \\
\hline III & 1.145 & $\begin{array}{l}0.645- \\
2.035\end{array}$ & 0.644 & III & 1.189 & $\begin{array}{l}0.667- \\
2.120\end{array}$ & 0.558 & III & 1.150 & $\begin{array}{l}0.646- \\
2.048\end{array}$ & 0.634 \\
\hline $\begin{array}{l}\text { The } \\
\text { prescription } \\
\text { dose }\end{array}$ & & & & $\begin{array}{l}\text { The } \\
\text { prescription } \\
\text { dose }\end{array}$ & & & & $\begin{array}{l}\text { The } \\
\text { prescription } \\
\text { dose }\end{array}$ & & & \\
\hline$<59.92$ Gy & 1 & & & $<59.92$ Gy & 1 & & & $<59.92$ Gy & 1 & & \\
\hline$\geq 59.92 \mathrm{~Gy}$ & 3.368 & $\begin{array}{l}1.323- \\
8.570\end{array}$ & 0.011 & $\geq 59.92 \mathrm{~Gy}$ & 3.348 & $\begin{array}{l}1.310- \\
8.556\end{array}$ & 0.012 & $\geq 59.92$ Gy & 3.849 & $\begin{array}{l}1.535- \\
9.652\end{array}$ & 0.004 \\
\hline LSV50 & & & & IliacV50 & & & & FemurV50 & & & \\
\hline Group1 & 1 & & & Group1 & 1 & & & Group1 & 1 & & \\
\hline Group2 & 1.463 & $\begin{array}{l}0.873- \\
2.452\end{array}$ & 0.049 & Group2 & 1.444 & $\begin{array}{l}0.849- \\
2.456\end{array}$ & 0.075 & Group2 & 1.044 & $\begin{array}{l}0.581- \\
1.877\end{array}$ & 0.886 \\
\hline \multicolumn{4}{|c|}{ Group1: < $111.41 \mathrm{ml}$; } & \multicolumn{4}{|c|}{ Group1: < 88.365 ml; } & \multicolumn{4}{|c|}{ Group1: < $1.23 \mathrm{ml}$; } \\
\hline \multicolumn{4}{|c|}{ Group2: $\geq 111.41 \mathrm{ml}$} & \multicolumn{4}{|c|}{ Group2: $\geq 88.365 \mathrm{ml}$} & \multicolumn{4}{|c|}{ Group2: $\geq 1.23 \mathrm{ml}$} \\
\hline
\end{tabular}

\section{Discussion}

As the development of the radiotherapy, the conventional radiotherapy technique has been gradually replaced by more advanced techniques such as IMRT, Vmat, and so on, which effectively reduced the volume and dose of irradiation of the normal tissue, but low dose irradiation volume did not decrease ${ }^{[5,6]}$, there are still many issues of concern. The concurrent chemoradiotherapy has become the standard treatment for locally advanced cervical cancer ${ }^{[4]}$. But meanwhile the hematological toxicity is still a common adverse event in the radiotherapy of pelvic tumor, when it is serious, it will limit the intensity of radiotherapy and chemotherapy, prolong the duration of the treatment, and ultimately lead to adverse impact to the treatment ${ }^{[7-9,20-22]}$. It has been found that up to $50 \%$ of a patient's total active bone marrow is within the pelvis and lumbar spine ${ }^{[23]}$. So it is important to protect the bone marrow of the pelvis and lumbar spine during the radiation of the pelvic tumor. The relationship between bone marrow exposure and hematological toxicity during the pelvic radiation has been proved, and reducing the radiation dose of bone marrow could reduce the risk of clinical hematological toxicity has although been proved ${ }^{[24,25]}$. The published results found that BM sparing IMRT could reduce acute hematological toxicity for patients with locally advance cervical cancer, the dosimetric parameters used were the mean dose, V10 and $\mathrm{V} 20^{[26,27]}$. But do every dosimetric parameter of different anatomical bone regions influence the hematological toxicity equally? Of course not. The hematopoietic function of bones depends on the content of their active bone marrow. Researchers have already proved the proportion of proliferating bone marrow varied in different anatomical sites of bones. ${ }^{[17-19]}$ Even in the pelvic skeleton, which includes the inferior lumbar vertebrae, the sacral vertebrae, the ilium and the upper femur, the radiation dose of these different 
anatomical regions influence the hematological/neutrophil toxicity differently. So in the process of setting the radiotherapy, the different anatomical skeletal regions should be treated differently as organs at risk.

In this research, we aimed to prove there were different correlation between different anatomical regions and the neutrophil toxicity. As our result showed, the mean dose of the LS was the highest, and the mean dose of the femur was the lowest; the volume of the high dose was the largest in the LS region, the volume of the low and medium dose was the largest in the ilium region, while all of the volume of dose in the femur region was negligible in compare with the other two regions. The LS vertebrae was close to the PTV/PGTV, so the volume of the high dose was higher than the other two regions, but the LS vertebrae is cylindrical bone, so in the cross sections, the area of the LS vertebrae was small, the isodose curves of low and medium dose avoided the LS in most conditions;

The ilium is flat bone, it is around the outside of the pelvic cavity, where the isodose curves of low and medium dose mostly distributed, so the volume of the low and medium dose of the ilium was the highest. The femur is almost out of the pelvic cavity, and is the farthest from the center of the target area of radiation, so all of the volume of dose in the femur region was negligible in compare with the other two regions. This might explained why the V20, V30 and V50 of the LS correlated well with the neutrophil toxicity, while the Dmean, V5, V10, V20 and V30 correlated well with the neutrophil toxicity, and none of the dosimetric parameters of the femoral correlated with the neutrophil toxicity. So we suggest that, in order to reduce the risk of neutrophil toxicity, the volume of medium and high dose of LS and the volume of low and medium dose of ilium should be strictly limited, the Dmean of the ilium should also be taken into consideration. While the dosimetric parameters of the femur could be ignored. However, this is a retrospective research, to get more precise results, the prospective randomized controlled study is needed.

\section{Conclusion}

During the process of concurrent chemoradiotherapy for cervical cancer, in order to reduce the risk of neutrophil toxicity, the volume of medium and high dose of LS and the volume of low and medium dose of ilium should be strictly limited, the Dmean of the ilium should also be taken into consideration. The dosimetric parameters of the femur could be ignored.

\section{Abbreviations}

EBRT

External-beam radiation therapy;

LS

Lumbosacral vertebrae;

IMRT

Intensity-modulated radiation therapy;

Vmat

Volume of intensity-modulated technology;

CTV

Clinical target volume;

GTVnd

Gross tumor target volume of lymph node;

PTV

Planning target volume;

PGTVnd

Planning target volume of GTVnd.

\section{Declarations}

\section{Ethics approval and consent to participate:}

This study was approved by the Regional Ethics Committee of Tianjin Medical University cancer institute and hospital and all patients were contacted by telephone to obtain verbal informed consent.

Page 13/19 


\section{Consent for publication:}

Not applicable.

\section{Availability of data and materials:}

The datasets analysed during the current study are available from the corresponding author on reasonable request.

\section{Competing interests:}

The authors declare that they have no competing interests.

\section{Funding:}

This study was supported by the National Natural Science Foundation of China (No. 81502656).

\section{Authors' contributions:}

Dr. Baozhong Zhang and Professor Liming Xu contributed equally to this work. Dr. Baozhong Zhang contributed to the work of patients' treatment, the follow-up, data collation, and article writing; Professor Liming Xu contributed to the work of the patients' treatment and data collation; Dr. Yanlan Chai, Dr. Yuanjie Cao, Pro. Hailing Hou, Dr. Jing Wang and Pro. Zhiyan Liu contributed to the work of the patients' treatment and follow-up.

\section{Acknowledgements:}

Not applicable.

\section{References}

1. Barakat R, Markman M, Randall M, editors. Principles and practice of gynecologic oncology. 5th ed. Philadelphia: Lippincott Williams \& Wilkins; 2009.

2. Nandakumar A, Ramnath T, Chaturvedi M. The magnitude of cancer cervix in India. Indian J Med Res. 2009;130:219-21.

3. Siegel R, Ma J, Zou Z, et al. Cancer statistics, 2014. CA Cancer J Clin. 2014;64:9Y29.

4. NCCN. National Comprehensive Cancer Network. Clinical Practice Guidelines in Oncology. 2015.

5. Mell LK, et al. Dosimetric comparison of bone marrow-sparing intensitymodulated radiotherapy versus conventional techniques for treatment of cervical cancer. Int J Radiat Oncol Biol Phys. 2008;71(5):1504-10.

6. Lujan $A E$, et al. Intensity-modulated radiotherapy as a means of reducing dose to bone marrow in gynecologic patients receiving whole pelvic radiotherapy. Int J Radiat Oncol Biol Phys. 2003;57(2):516-21.

7. Green JA, et al. Survival and recurrence after concomitant chemotherapy and radiotherapy for cancer of the uterine cervix: a systematic review and metaanalysis. Lancet. 2001;358(9284):781-6.

8. Torres MA, et al. Comparison of treatment tolerance and outcomes in patients with cervical cancer treated with concurrent chemoradiotherapy in a prospective randomized trial or with standard treatment. Int J Radiat Oncol Biol Phys. 2008;70(1):11825.

9. Abu-Rustum NR, et al. Compliance with and acute hematologic toxic effects of chemoradiation in indigent women with cervical cancer. Gynecol Oncol. 2001;81(1):88-91.

10. Mell LK, et al. Dosimetric predictors of acute hematologic toxicity in cervical cancer patients treated with concurrent cisplatin and intensity-modulated pelvic radiotherapy. Int J Radiat Oncol Biol Phys. 2006;66(5):1356-65. 
11. Rose BS, et al. Normal tissue complication probability modeling of acute hematologic toxicity in cervical cancer patients treated with chemoradiotherapy. Int J Radiat Oncol Biol Phys. 2011;79(3):800-7.

12. Ahmed RS, Kim RY, Duan J, et al. IMRT dose escalation for positive para-aortic lymph nodes in patients with locally advanced cervical cancer while reducing dose to bone marrow and other organs at risk. Int J Radiat Oncol Biol Phys. 2004;60:505-12.

13. Brixey CJ, Roeske JC, Lujan AE, et al. Impact of intensitymodulated radiotherapy on acute hematologic toxicity in women with gynecologic malignancies. Int J Radiat Oncol Biol Phys. 2002;54:1388-96.

14. Chen MF, Tseng CJ, Tseng CC, et al. Clinical outcome in posthysterectomy cervical cancer patients treated with concurrent Cisplatin and intensity-modulated pelvic radiotherapy: Comparison with conventional radiotherapy. Int J Radiat Oncol Biol Phys. 2007;67:1438-44.

15. Hong L, Alektiar K, Chui C, et al. IMRT of large fields: wholeabdomen irradiation. Int J Radiat Oncol Biol Phys. 2002;54:278-89.

16. Hanbyoul Cho B-H, Nam SM, Kim, et al. A Phase 2 Trial of Radiation Therapy With Concurrent Paclitaxel Chemotherapy After Surgery in Patients With High-Risk Endometrial Cancer: A Korean Gynecologic Oncologic Group Study. International Journal of Radiation Oncology Biology Physics May 16th. 2014;90(1):140-6.

17. Sherley JL, Kelly TJ. Regulation of human thymidine kinase during the cell cycle. J Biol Chem. 1988;263:8350-8.

18. Everitt S, Hicks RJ, Ball D, et al. Imaging cellular proliferation during chemo-radiotherapy: A pilot study of serial $18 \mathrm{~F}-\mathrm{FLTPET} / \mathrm{CT}$ imaging for non-small-cell lung cancer. Int J Radiat Oncol Biol Phys. 2009;75:1098-104.

19. DISTRIBUTION OF PROLIFERATING BONE MARROW IN ADULT CANCER PATIENTS DETERMINED USING FLT-PET IMAGING HAYMAN JAMESA, CALLAHAN JASONW, et aI, DISTRIBUTION OF PROLIFERATING BONE MARROW IN ADULT CANCER PATIENTS DETERMINED USING FLT-PET IMAGING, Int. J. Radiation Oncology Biol. Phys., Volume 79, Number 3, 2011.

20. Klopp AH, Moughan J, Portelance L, et al. Hematologic toxicity in RTOG 0418: a phase 2 study of postoperative IMRT for gynecologic cancer. Int J Radiat Oncol Biol Phys. 2013;86:83-90.

21. Bazan JG, Luxton G, Mok EC, Koong AC, Chang DT. Normal tissue complication probability modeling of acute hematologic toxicity in patients treated with intensity-modulated radiation therapy for squamous cell carcinoma of the anal canal. Int $J$ Radiat Oncol Biol Phys. 2012;84:700-6.

22. Shaikh T, Wang L, Egleston BL, Burki M, Hoffman JP, Cohen SJ, et al. Predictors of hematologic toxicity and chemotherapy dose intensity in patients undergoing chemoradiation for pancreatic cancer. [published online August 28, 2015]. Am J Clin Oncol.

23. Rose BS, et al. Correlation between radiation dose to (1)(8)F-FDG-PET defined active bone marrow subregions and acute hematologic toxicity in cervical cancer patients treated with chemoradiotherapy. Int J Radiat Oncol Biol Phys. 2012;83(4):118591.

24. Yovino S, Kleinberg L, Grossman SA, et al. The etiology of treatment-related lymphopenia in patients with malignant gliomas: Modeling radiation dose to circulating lymphocytes explains clinical observations and suggests methods of modifying the impact of radiation on immune cells. Cancer Investig. 2013;31:140-4.

25. Mell LK, Kochanski JD, Roeske JC, et al. Dosimetric predictors of acute hematologic toxicity in cervical cancer patients treated with concurrent cisplatin and intensity-modulated pelvic radiotherapy. Int J Radiat Oncol Biol Phys. 2006;66:1356-65.

26. Mell LK, et al. Bone marrow-sparing intensity modulated radiation therapy with concurrent cisplatin for stage IB-IVA cervical cancer: an international multicenter phase II clinical trial (INTERTECC-2). Int J Radiat Oncol Biol Phys. 2017;97(3):536-45.

27. Nan Li SS, Noticewala, Casey W, Williamson, et al. Feasibility of atlas-based active bone marrow sparing intensity modulated radiation therapy for cervical cancer. Radiother Oncol. 2017;123:325-30.

\section{Figures}




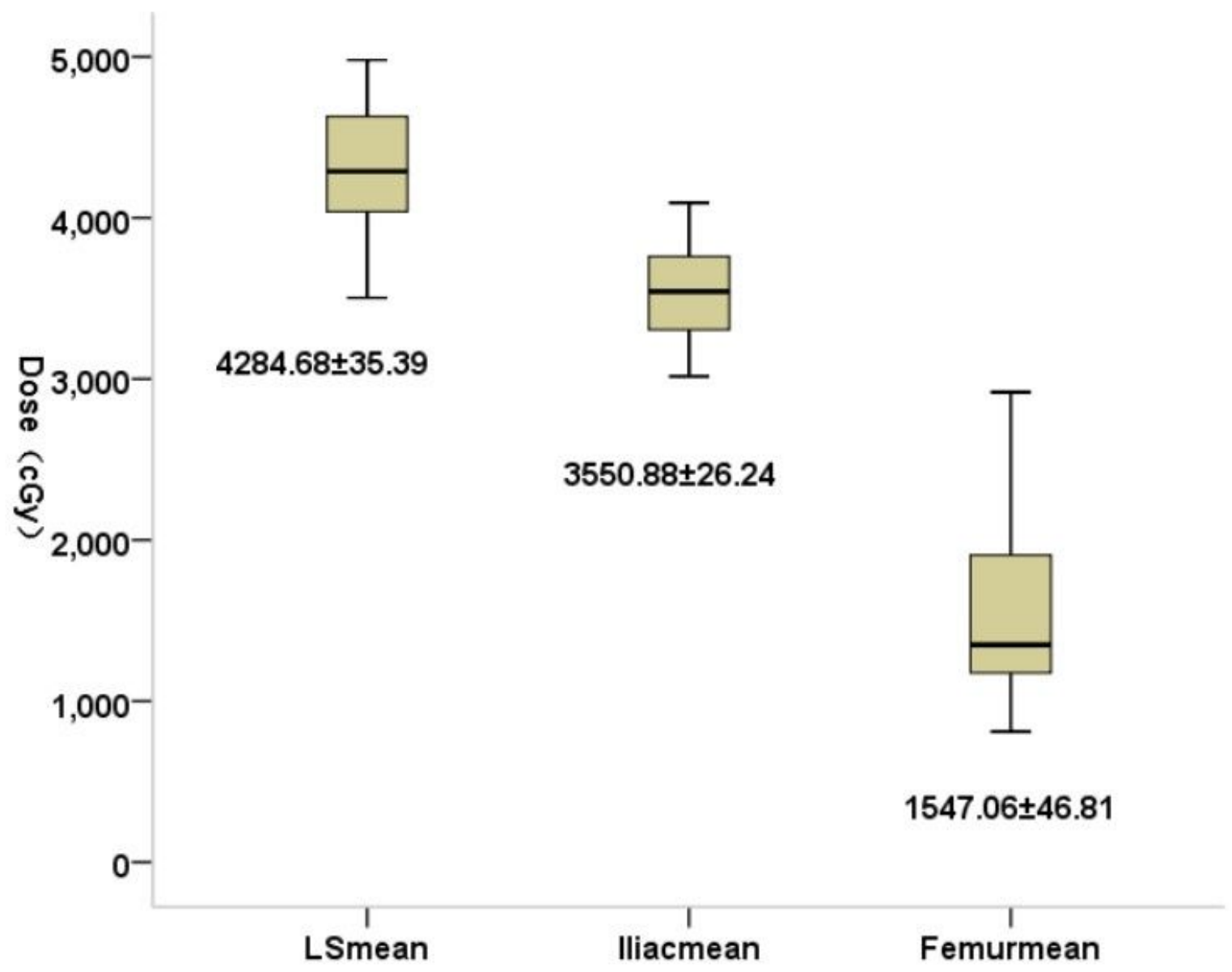

Figure 1 


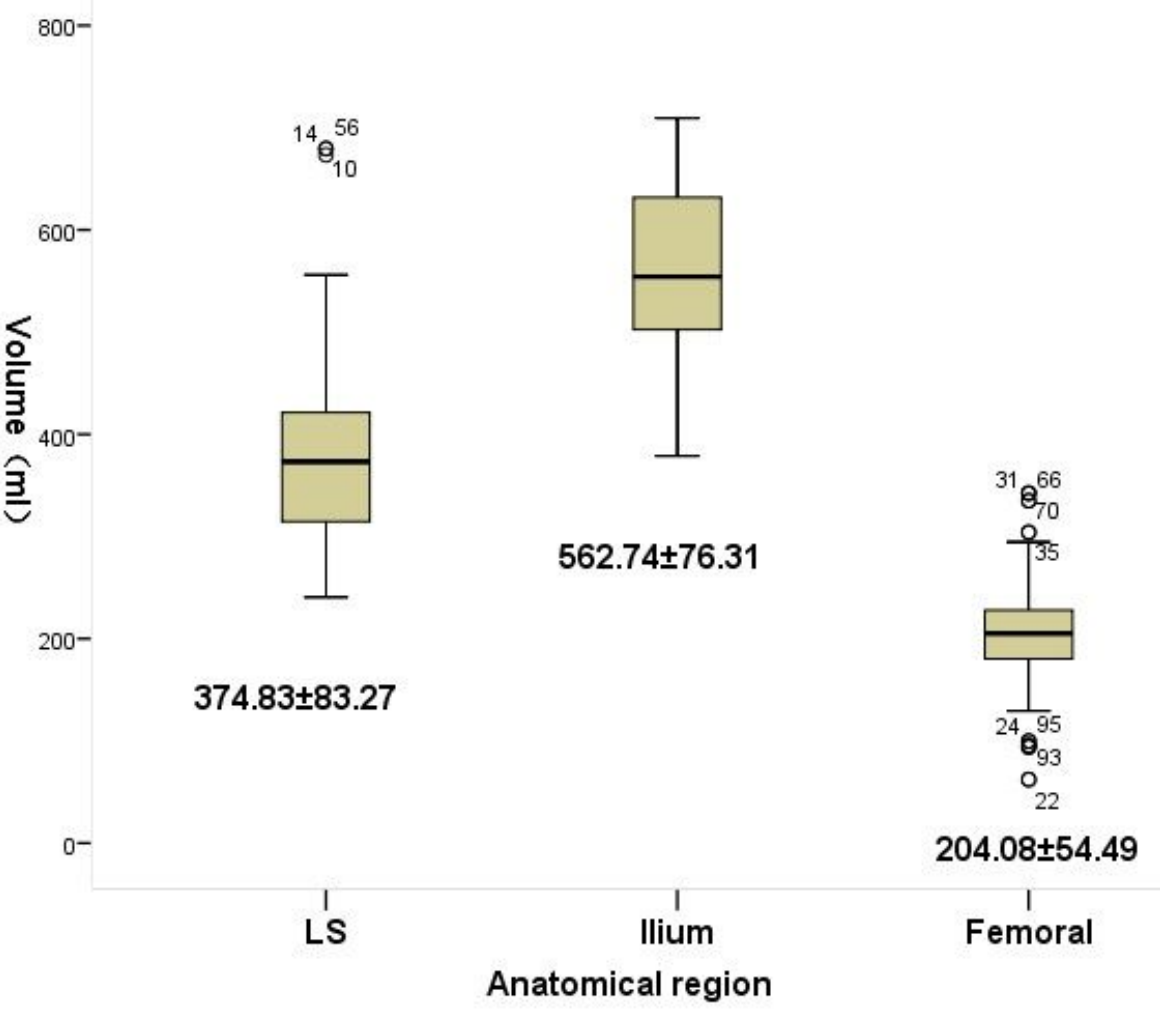

Figure 2

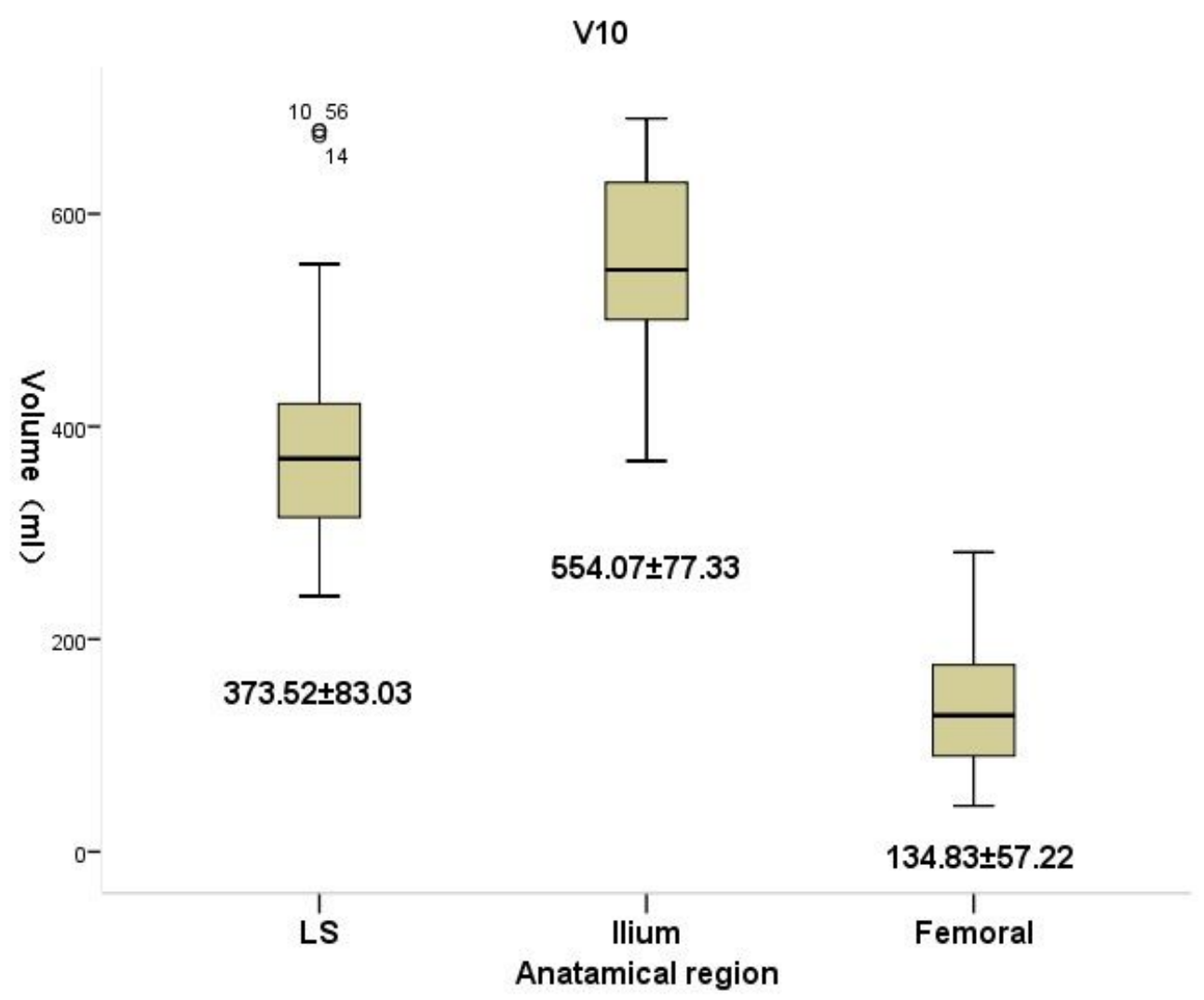

Figure 3 


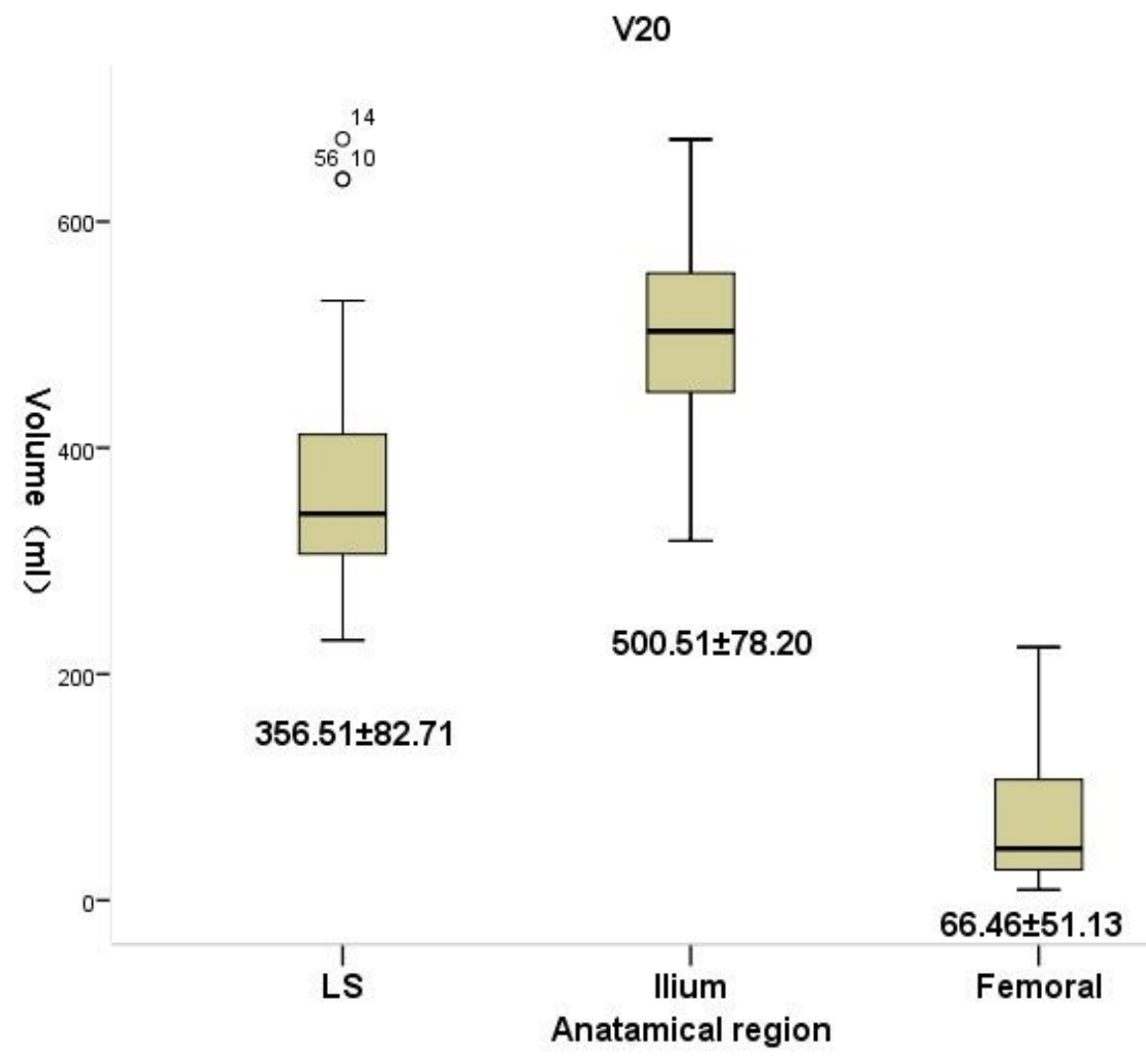

Figure 4

V30

$$
0^{14}
$$

600-

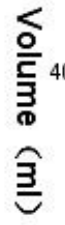

$200^{-}$
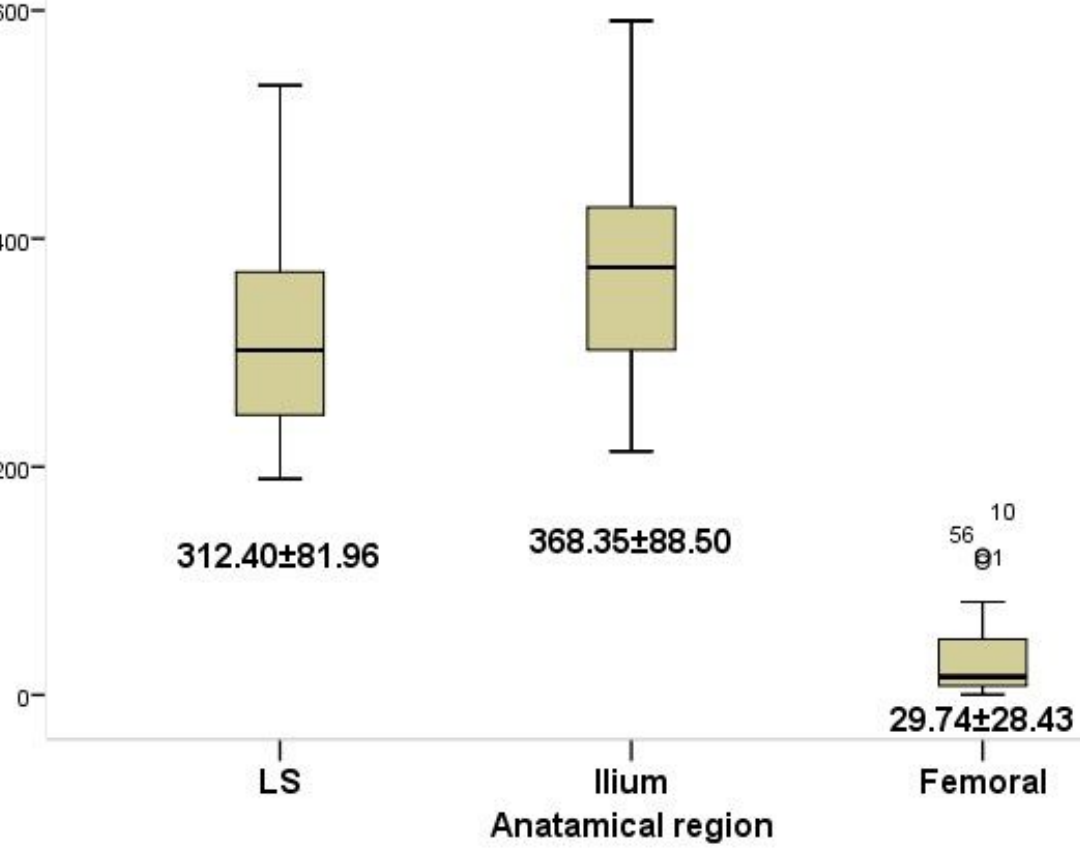

Figure 5 


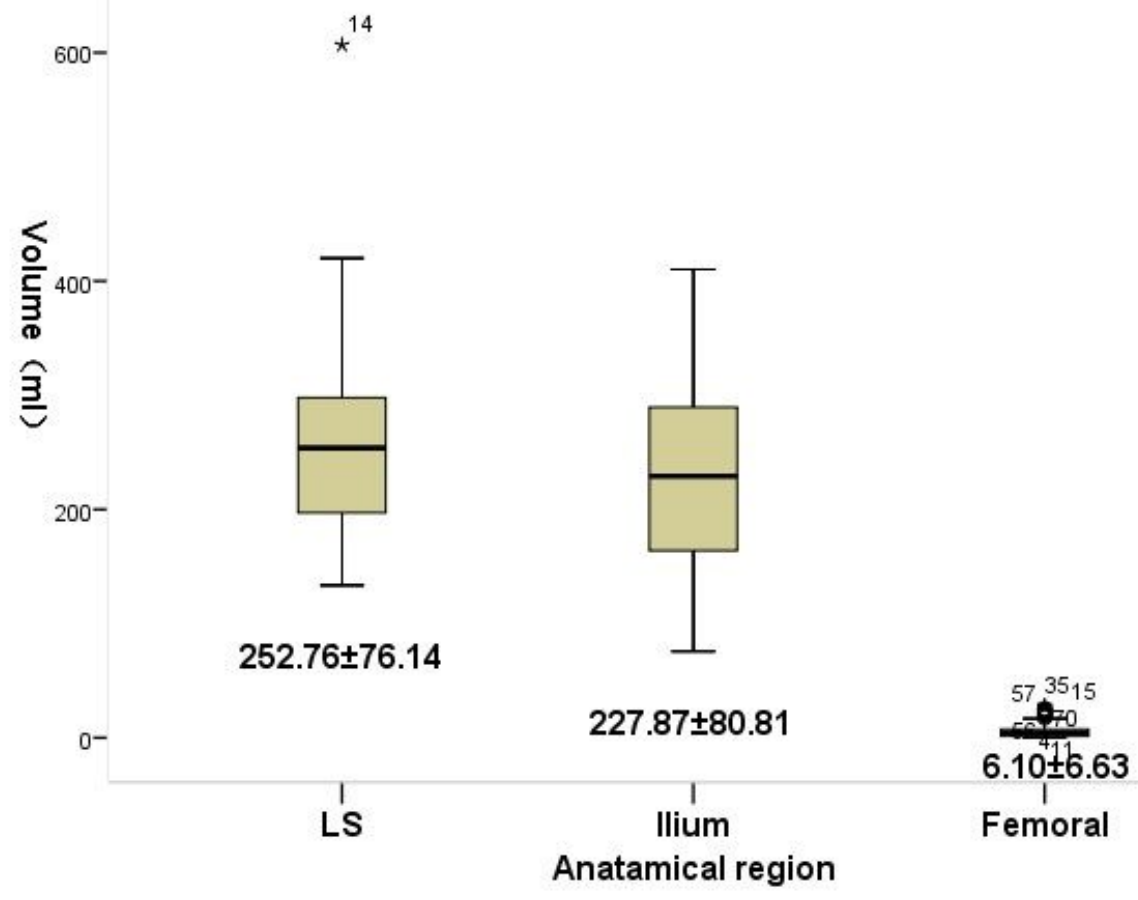

Figure 6

V50

400-

$300^{-}$

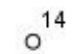

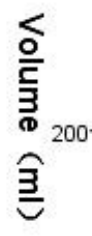

$100-$

0-

$$
0^{14}
$$

Figure 7

Page 19/19 